\title{
SURGICAL STABILISATION OF THE SPINE
}

\author{
By R. W. JACKSON, M.D., M.S., F.R.C.S.(C) \\ Division of Orthopaedic Surgery, Toronto General Hospital, Toronto, Canada
}

THE primary purpose of this paper is to acquaint the non-surgical members of the Society with some of the indications and methods used by orthopaedic surgeons for the stabilisation of the vertebral column after trauma. Most orthopaedists agree that one should consider both the immediate and the future stability of the vertebral column at the time the initial neurological appraisal of the patient is carried out. The orthopaedist's responsibility in the team management of the patient is to ensure that painless stability is the eventual result of treatment of the vertebral column. However, he must do this with careful regard for the general condition of the patient, and the specific condition of the spinal cord.

Guttmann has constantly stressed that decompressive laminectomy is rarely, if ever, of any value in the management of an acute spinal cord injury (Frankel et al., 1969; Guttmann, 1973). This message has generally reached most parts of the world. There are still however, two types of physicians who are at opposite ends of the surgical spectrum and to whom this paper is also directed. At one end are those who have somewhat over-reacted to Guttman's teachings and who. feel that decompression is synonymous with surgery, and who therefore advocate no surgery whatsoever. At the other end are those who disregard his teachings, who still perform extensive laminectomies, and who thereby grossly compound the problems of stabilisation at a later date.

\section{PRINCIPLES OF ORTHOPAEDIC TREATMENT}

The orthopaedist is guided by several well-established principles in his treatment of trauma to the vertebral column. The first principle is that a disruption which primarily involves the bony structures of the vertebral column will heal by bone, and will be quite stable once healing has occurred. A simple example of this is the wedge compression fracture of the vertebral body in which the posterior elements are intact. Another example is the bursting type of fracture involving the body, or fractures of the laminae or pedicles-such as the typical 'hangman's fracture', which involves the posterior elements of $\mathrm{C}_{2}$. These lesions will heal by bone and do not require surgical stabilisation (Holdsworth, I970). However, they do require a period of relative immobilisation during the healing phase. It is estimated that more than 90 per cent of injuries to the spinal column are inherently stable or will become stable in time, and therefore do not require surgical stabilisation (Chesire, 1969; Frankel, 1969; Bedbrook, I970; Bedbrook \& Ebidan, 1973).

The second principle is that a disruption which occurs with minimal bony damage, but with gross tearing of ligaments, capsule and other soft tissue structures, tends to heal by fibrous scar tissue which can stretch, and is therefore a potentially unstable lesion (Holdsworth, 1970). Common examples of such potentially unstable lesions are facet dislocations, rotatory fracture dislocations as described by I3/2-A* 
Holdsworth (I970), and CI, 2 subluxations due to tearing of the transverse ligament, rather than fracturing of the odontoid.

The third principle which has been well proven by Guttmann (1973) and others (Frankel, 1969; Bedbrook, 1970; Braakman \& Penning, 1973) is that surgical decompression through laminectomy, is rarely, if ever, of any value to the traumatised spinal cord, and indeed may be extremely detrimental to the spinal column. This does not require further elaboration.

\section{INDICATIONS FOR SURGERY}

The orthopaedic indications for surgery are therefore based on these three principles, and are intended to produce a painless, stable and balanced vertebral column at the conclusion of treatment. A decision to surgically stabilise a spine is made independently of neurological considerations, but is only carried out when the neurological state is optimal for the procedure. Usually, this is in the subacute stage, seven to ten days after injury (or even later), when oedema and inflammation have subsided, the patient's general condition has stabilised and there is less risk of further damage to the cord.

(a) Early Indications. One must again stress that early indications for stabilising surgery in the presence of a severe neurological deficit are extremely rare. A review of 428 cases of potentially unstable flexion-rotation injuries of the cervical spine revealed only 20 cases $(4.6$ per cent) which showed late instability (Bedbrook, 1970). On the other hand, two advantages of stabilising surgery are a reduction in the degree of deformity after union, and significantly less discomfort during the early stages of treatment (Bedbrook \& Ebidan, I973; Weiss, I973).

Fusion for potential instability in the patient with an incomplete lesion or an undamaged cord might well be justified on the basis of protecting the patient against possible serious cord damage from relatively minimal trauma in the future. If considered necessary, such surgery should be done at the optimal time.

Early fusion or stabilisation for gross instability would be necessary only in those few instances, where conservative measures cannot be adequately carried out due to other injuries, such as severe chest problems, burns or multiple extremity injuries.

The dislocation that is still unreduced after adequate conservative management should be reduced atraumatically by surgery. Again, while the decision to operate is made independently of the neurological state, the actual surgery is performed at the optimal time when there is the least chance of an adverse effect on the cord. That is to say, the time of surgery is very dependent upon the neurological state. Minimal bone should be taken to avoid any further trauma to the cord. Internal fixation devices should be used for immediate stability, and autogenous bone grafts also employed for ultimate stability.

(b) Late Indications. The late indications for surgery become apparent after maximum neurological recovery has occurred, and when the patient attempts to sit or otherwise mobilise. The indications are usually pain due to instability, or progressive deformity. Instability may be detected by bending films or by tomography showing failure of solid bony union at the suspected site. Pain may result from the lowest surviving nerve roots being compressed or irritated as they leave the vertebral foramina. 
Another indication is the correction of deformity. If the vertebral column is markedly unstable, an increasing kyphosis may develop as attempts are made to sit or ambulate. Spinal osteotomy, internal fixation and bone grafting is then necessary for a stable and balanced spine (Jackson \& Langer, I973).

Another type of post-traumatic deformity is the collapsing spine secondary to a high lesion or to an ascending myelopathy of the cord with progressive loss of the supporting paraspinal musculature. Again, surgical correction of the deformity and stabilisation by internal fixation plus bone grafting can produce excellent end results (Jackson, I966).

\section{INTERNAL FIXATION DEVICES}

Of the various fixation devices that are commonly used, wire of a fairly heavy gauge is still the most common. Other stabilising devices which can be used in a variety of situations, include plates, springs, clamps and rods.

Two types of onlay metal plates have gained some prominence over the years. These are the Meurig Williams spinal plates, and the Wilson plates. Ideally, one should obtain a firm grip on at least two spinous processes above and two below the level of involvement. If bone graft is also placed at the level of involvement, it is possible to electively remove the plates 12 months or more later, and once

again permit movement to occur at the uninvolved levels.

In recent years, Harrington instrumentation has proven useful in stabilising both scoliotic and traumatised spines. A combination of distraction rods and compression hooks can be used, with purchase being obtained on laminae, transverse processes, facet joints, or even ribs (Bedbrook \& Ebidam, 1973; Jackson \& Langer, 1973).

Weiss has developed a unique method using springs placed on either side of the spinous processes, and hooked into the laminae. His results in both stabilising the unstable spine and in gently correcting a deformity are most encouraging (Weiss, 1973).

\section{BONE GRAFTING}

As a general rule autogenous bone should be used as graft material, to reinforce any mechanically stabilised spine. One cannot rely on metal fixation to maintain a reduction indefinitely-bony union must be obtained to achieve true stability. Some bone grafting techniques by themselves can provide a degree of mechanical stability, as well as promote osteogenesis.

If an anterior approach to the spinal column is necessary, the graft material is usually cortico-cancellous and is inlaid between vertebral bodies.

If a posterior approach is used, tibial grafts or cortico-cancellous iliac grafts can be used in an onlay fashion.

Of increasing clinical value in the treatment of lumbar lesions is the lateral fusion, where cortico-cancellous grafts are laid from one transverse process to another, thus bridging the necessary spaces. The grafts mature well, as they lie in an ideal bed, surrounded by muscle-but no stability is afforded.

An anterior approach is necessary when an extensive posterior decompression has removed all bony elements to which metallic fixation devices could be attached, or when it is necessary to 'jack-up' an unstable and dislocated segment. 
If a posterior approach is possible and desirable cortical bone grafts may be affixed to the spinous processes for both immediate and ultimate stability. If metallic devices are used for immediate stability, a mixture of cortical and cancellous bone graft material can be placed between the transverse processes and around the posterior elements of the involved vertebrae. When these grafts are mature, the metal can be removed.

\section{SUMMARY}

It is important to assess spinal injuries, both in terms of cord damage and column damage, shortly after the trauma has occurred. A decision should then be made concerning the eventual stability of the bony vertebral column. If a lesion is unlikely to heal soundly by bone or if one is unable to obtain a reduction by conservative measures, one should consider surgical stabilisation of the damaged spine in the subacute phase. At the same time as one stabilises the spine with metallic fixation, bone grafts should be added for ultimate stability. If an unstable spine is encountered in the later stages of treatment, surgical stabilisation is again indicated. Metallic fixation can greatly aid in stabilising the spine until bone grafts can mature. Osteotomies of the spine through a combined transthoracic and posterior approach can be extremely valuable in correcting deformity.

While there is no role for decompressive surgery after spinal cord injury, there is still a definite role for surgery in balancing and stabilising the vertebral column.

\section{REFERENCES}

Bedbrook, G. M. \& Edibam, R. C. (1973). The study of spinal deformity in traumatic spinal paralysis. Int. F. of Paraplegia, 10, 321-335.

Bedbrook, G. M. (1970). Results in Spinal Paralysis Presented at a Post-Convention Meeting of the Fifth Combined Meeting of the English Speaking Orthopaedic Associations.

Braakman, R. \& Penning, L. (1973). Mechanisms of injury to the cervical cord. Int. $\mathcal{F}$. Paraplegia, 10, 314-320.

ChesiRE, D. J. E. (I969). The stability of the cervical spine following the conservative treatment of fractures and fracture-dislocations. Int. F. of Paraplegia, 7, 193-203.

FRANKEL, H. L. et al. (I969). The value of postural reduction in the initial management of closed injuries of the spine with paraplegia and tetraplegia. Part I. Int. F. Paraplegia, 7, I79-192.

GutTMAn, SIR LudWIG (1973). Spinal Cord Injuries-Comprehensive Management and Research. Blackwell Scientific Publications Ltd.

HolDSwORTH, F. W. (I970). Fractures, dislocations and fracture-dislocations of the spine. F. Bone E foint Surg. 52A, I 534-I 55 I.

JACKSON, R. W. \& LANGER, B. (I973). Spinal osteotomy to correct deformity in paraplegia. Modern Medicine, 28, 663-665.

JACKSON, R. W. (I966). Correction of spinal curvature in paraplegics by the method of Harrington fusion. Medical Services fournal, Canada, 22, 486-495.

Weiss, M. (I973). Personal communication. 